\title{
In vivo real-time imaging of chemotherapy response on the liver metastatic tumor microenvironment using multiphoton microscopy
}

\author{
KOJI TANAKA ${ }^{1}$, MASATO OKIGAMI ${ }^{1}$, YUJI TOIYAMA ${ }^{1}$, YUKI MORIMOTO ${ }^{1}$, \\ KOHEI MATSUSHITA ${ }^{1}$, MIKIO KAWAMURA ${ }^{1}$, KIYOSHI HASHIMOTO ${ }^{1}$, \\ SUSUMU SAIGUSA $^{1}$, YOSHINAGA OKUGAWA ${ }^{1}$, YASUHIRO INOUE ${ }^{1}$, KEIICHI UCHIDA ${ }^{1}$, \\ TOSHIMITSU ARAKI ${ }^{1}$, YASUHIKO MOHRI ${ }^{1}$, AKIRA MIZOGUCHI ${ }^{2}$ and MASATO KUSUNOKI ${ }^{1}$ \\ Departments of ${ }^{1}$ Gastrointestinal and Pediatric Surgery, and ${ }^{2}$ Neural Regeneration and \\ Cell Communication, Mie University Graduate School of Medicine, Tsu, Mie 514-8507, Japan
}

Received June 12, 2012; Accepted August 3, 2012

DOI: 10.3892/or.2012.1983

\begin{abstract}
In vivo real-time visualization of chemotherapy response at the cellular level provides us with direct evidence of what happens on the tumor microenvironment of metastatic organs. We imaged the response of metastatic tumor cells and host stromal cells to chemotherapeutics on liver metastatic xenografts in living mice using intravital twophoton laser scanning microscopy (TPLSM). Red fluorescent protein-expressing human colorectal cancer cells (HT29) was inoculated to the spleen of green fluorescent proteinexpressing nude mice. 5-Fluorouracil or irinotecan was intraperitoneally administered after the formation of macroscopic liver metastases. Intravital TPLSM was performed at multiple time-points for time-series imaging of liver metastatic xenografts in the same mice. Under the 1st TPLSM, HT29 cells were visualized in hepatic sinusoids at the single cell level. Liver metastatic nodules consisting of viable cancer cells and surrounding stroma with tumor vessels were visualized under the 2nd TPLSM. After chemotherapy, tumor cell fragmentation, condensation, swelling and intracellular vacuoles were observed under the 3rd TPLSM. There was no obvious morphological difference in tumor response between these chemotherapeutics. Time-series intravital TPLSM imaging on the metastatic tumor xenografts may be useful for screening and evaluating new chemotherapeutics with less interindividual variability.
\end{abstract}

Correspondence to: Dr Koji Tanaka, Department of Gastrointestinal and Pediatric Surgery, Mie University Graduate School of Medicine, 2-174 Edobashi, Tsu, Mie 514-8507, Japan

E-mail: qouji@clin.medic.mie-u.ac.jp

Key words: colorectal cancer, liver metastasis, tumor microenvironment, multiphoton microscopy, intravital imaging

\section{Introduction}

In the treatment of metastatic colorectal cancer (mCRC), systemic chemotherapy comprising 5-fluorouracil plus leucovorin combined with oxaliplatin (FOLFOX), or irinotecan (FOLFIRI) in addition to molecular targeted agents, bevacizumab or cetuximab or panitumumab, has achieved higher response rates and prolonged patient survival $(1,2)$. These chemotherapy regimens have increased the conversion rates of initially unresectable metastases to resectable ones in patients with mCRC. Secondary resection following the downsizing of unresectable metastases by chemotherapy has provided not only the survival prolongation but also potential cure $(3,4)$. Thus, chemotherapy response for metastatic diseases plays an important role on treatment decision-making of mCRC patients, because it is one of the most predictive for overall survival of such patients (3-5).

The molecular mechanisms of the above mentioned chemotherapeutics have been explored by either in vitro or in vivo studies (6-8). It still remains important to understand how metastatic tumor cells and/or stromal cells respond to chemotherapy on the metastatic organs, because the prognosis of cancer patients depends on metastatic disease control. However, there is no study of in vivo real-time visualization of chemotherapy response at the cellular level on the microenvironment of metastatic tumor in living animals.

Multiphoton microscopy including two-photon laser scanning microscopy (TPLSM) has been widely used and become an indispensable tool in the field of tumor biology $(9,10)$. TPLSM has made it possible to analyze the structural and functional changes at the single cell level because of the ability of high resolution and high magnification imaging. It has also extended intravital imaging at a deeper tissue level with minimal phototoxicity and photobleaching to living cells, compared with single-photon confocal laser scanning microscopy $(11,12)$. Intravital TPLSM imaging has enabled us to study the dynamics of cellular interactions in either two-dimensional (time-lapse) or three-dimensional (z-stacks) environment for long time in living animals. 
We have established a method for in vivo real-time TPLSM imaging of intra-abdominal gastrointestinal disease using green fluorescent protein (GFP)-expressing mice (13-17). Intravital TPLSM imaging with high resolution and high magnification was accomplished by the reduction of motion artifact due to cardiac and respiratory movement using an organ stabilizing system (Japanese patent application number, P2007-129723). Time-series (at multiple time points) intravital TPLSM imaging in the same mice over the long experimental periods was also accomplished by the prevention of abdominal adhesions using a sodium hyaluronate and carboxymethylcellulose membrane.

In the present study, we visualized in vivo real-time chemotherapy response for metastatic tumor xenografts using intravital TPLSM to study the dynamic interactions between metastatic tumor cells and host stromal cells on the microenvironment of metastatic organs in living animals.

\section{Materials and methods}

GFP-expressing nude mice. GFP-expressing nude mice (C57BL/6-BALB/c-nu/nu-EGFP) were purchased from AntiCancer Japan (Osaka, Japan). GFP nude mice (20-22 g) were bred, housed in groups of six mice per cage and fed with a pelleted basal diet (CE-7, CLEA Japan Inc., Tokyo, Japan). Mice had free access to drinking water. They were kept in the animal house facilities at Mie University School of Medicine under standard conditions of humidity $(50 \pm 10 \%)$, temperature $\left(23 \pm 2^{\circ} \mathrm{C}\right)$ and light $(12 / 12 \mathrm{~h}$ light/dark cycle), according to the Institutional Animal Care Guidelines. The experimental protocols were reviewed and approved by the Animal Care and Use Committee at Mie University Graduate School of Medicine.

RFP-expressing human CRC cell line. The RFP-expressing human CRC cell line (RFP-HT29) was purchased from AntiCancer Japan. RFP-HT29 cells were grown in monolayer cultures in RPMI-1640 (Sigma-Aldrich, Inc., St. Louis, MO, USA) supplemented with fetal bovine serum (10\% (v/v), Gibco BRL, Tokyo, Japan), glutamine (2 mM), penicillin (100,000 U/l), streptomycin $(100 \mathrm{mg} / \mathrm{l})$, and gentamycin $(40 \mathrm{mg} / \mathrm{l})$ at $37^{\circ} \mathrm{C}$ in a $5 \% \mathrm{CO}_{2}$ environment. For routine passage, cultures were spilt 1:10 when they reached $90 \%$ confluence, generally every 3 days. Cells at the fifth to ninth passage were used for liver metastasis experiments, which were performed with exponentially-growing cells.

Chemotherapeutic drugs. 5-Fluorouracil (5-FU) and irinotecan (CPT-11) were purchased from Sigma-Aldrich, Inc. The stock solutions of these drugs were made in dimethylsulfoxide (DMSO) and then were dissolved in appropriate concentrations with distilled water for in vivo study. To image the response to 5-FU or CPT-11 for liver metastatic xenografts, $5-\mathrm{FU}(50 \mathrm{mg} / \mathrm{kg})$ or CPT-11 (20 mg/kg) was administered intraperitoneally three times a week for three weeks.

Murine liver metastasis model. RFP-HT29 cells were inoculated into the spleens of GFP nude mice, as a colorectal liver metastatic xenograft model. RFP-HT29 cells at the fifth to ninth passage were harvested with trypsin/EDTA, and washed in serum-containing RPMI-1640 medium to inactivate any remaining trypsin. The cells were centrifuged and resuspended in phosphate-buffered saline (PBS). Finally, the cells were adjusted to $2 \times 10^{7}$ cells $/ \mathrm{ml}$ for single cell suspensions. GFP nude mice were initially anesthetized by intraperitoneal injection of chloral hydrate (Sigma). Under direct vision, $2 \times 10^{6}$ cells were injected into the spleen using a 30 -gauge needle through a small incision in the left lateral abdomen of anesthetized GFP nude mice.

Liver stabilization for intravital TPLSM. After inoculation, GFP nude mice were initially anaesthetized using an anaesthetic mask with $4 \mathrm{l} / \mathrm{min}$ of isofluorane (4\%; Forane, Abbott, Japan). Anaesthetic maintenance was achieved using 1.5-2\% isoflurane and $4 \mathrm{l} / \mathrm{min}$ of $\mathrm{O}_{2}$. Body temperature was kept at $37^{\circ} \mathrm{C}$ throughout the experiments using a heating pad. Normal saline (200 $\mu \mathrm{l}$ ) was administered intraperitoneally at 1-2 h intervals for hydration during anesthesia. The upper midline laparotomy was made as short as possible $(<15 \mathrm{~mm})$. The left lateral lobe of the liver was identified and exteriorized through the laparotomy. The liver lobe was then put onto an organ-stabilizing system (Japanese patent application number, P2007-129723) using a solder $1 \mu \mathrm{g}$ terminal with an instant adhesive agent (KO-10-p20, Daiso, Japan). The organ stabilizer minimized the microvibration of the observed area caused by heart beat and respiratory movements.

Stabilization and fixation of the liver lobe represented a critical but technically difficult part of the intravital TPLSM procedure. After the application of PBS to the observed area, a thin cover glass was placed gently on the liver surface. After intravital TPLSM, the exteriorized liver lobe was gently removed from the organ-stabilizing system using a release agent (KO-10-p8, Daiso), to prevent liver injury. The liver surface were extensively washed by PBS to remove a release agent and blood coagulation mass. A sodium hyaluronate and carboxymethylcellulose membrane (Seprafilm Adhesion Barrier, Genzyme Corporation, Cambridge, MA) was placed between the liver and the abdominal wall to prevent postoperative dense adhesion.

TPLSM setup. The procedures for TPLSM setup were performed as previously described (16). Experiments were performed using an upright microscope (BX61WI; Olympus, Tokyo, Japan) and an FV1000-2P laser-scanning microscope system (Fluoview FV1000MPE, Olympus). The use of special stage risers enabled the unit to have an exceptionally wide working distance. This permitted the stereotactically immobilized, anesthetized mouse to be placed on the microscope stage. The microscope was fitted with several lenses with high numeric apertures to provide the long working distances required for in vivo work, and with water-immersion optics. The excitation source in TPLSM mode was Mai Tai Ti:sapphire lasers (Spectra Physics, Mountain View, CA), tuned and modelocked at $910 \mathrm{~nm}$. The Mai Tai produces light pulses of $100 \mathrm{fs}$ width (repetition rate $80 \mathrm{MHz}$ ). Laser light reached the sample through the microscope objectives, connected to an upright microscope (BX61WI; Olympus). A mean laser power at the sample was between 10 and $40 \mathrm{~mW}$, depending on the depth of imaging. Microscope objective lens used in this study were 4xUPlanSApo (numerical aperture of 0.16), 10xUPlanSApo (numerical aperture of 0.4), and 60xLUMPlanFI/IR (water dipping, numerical aperture of 0.9 , working distance $2 \mathrm{~mm}$ ), respectively. 


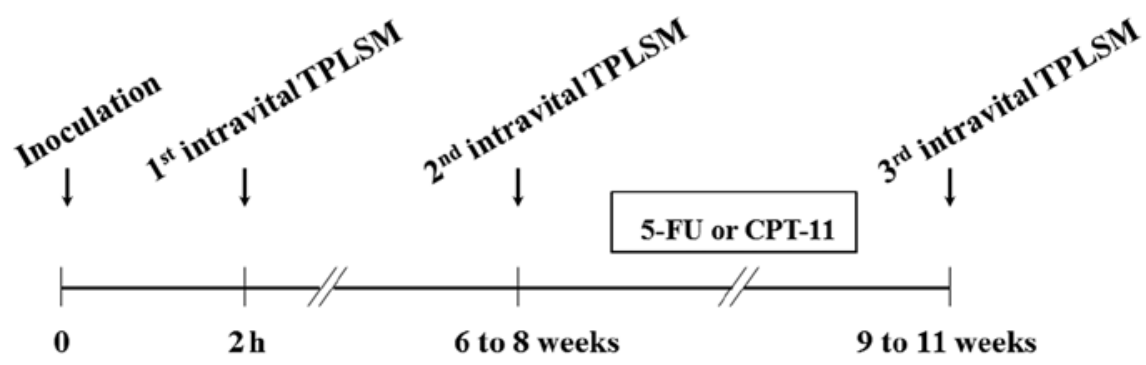

Figure 1. The timing of intravital TPLSM imaging. The 1st intravital TPLSM at $2 \mathrm{~h}$ after the successful inoculation. The 2nd inrtavital TPLSM in the same mice at 6-8 weeks after inoculation for the observation of the liver metastatic formation and their tumor microenvironment. In tumor bearing mice, 5-FU $(50 \mathrm{mg} / \mathrm{kg})$ or CPT-11 $(20 \mathrm{mg} / \mathrm{kg})$ was administered intraperitoneally three times a week for three weeks. The 3rd intravital TPLSM in the same mice at 9-11 weeks after inoculation for the visualization of in vivo real-time chemotherapy response on the tumor microenvironment of liver metastatic xenografts.

Data were analyzed using the FV10-ASW system (Olympus). TPLSM images were acquired with 512x512 pixels spatial resolution, from $210 \mu \mathrm{m}$ field of view dimension, using a pixel dwelling time $4 \mu$ sec. Two-photon fluorescence signals were collected by an internal detector (non-descanned detection method) at an excitation wavelength, to enable the simultaneous acquisition of EGFP signal and RFP (DsRed2) signal. An excitation wavelength of $1050 \mathrm{~nm}$ is optimum for DsRed2 as reported by Kawano et al (18), although an excitation wavelength of $910 \mathrm{~nm}$ is optimum for EGFP. Therefore, it is difficult to amply excite DsRed2 at 910-nm. To overcome this difficulty, we have selected and used the tumor cells in which expression level of DsRed2 is so high that we can identify the DsRed2 labeled tumor cells clearly even with 910-nm excitation. Colorcoded green and red images were taken at the same time, and subsequently merged to produce single images.

Imaging methods using TPLSM. The surface of the liver lobe was initially screened at lower magnifications by setting out the $\mathrm{X} / \mathrm{Y}$ plane and adjusting the $\mathrm{Z}$ axis manually to detect the optimal observation area containing RFP-expressing cancer cells (at least five areas). Each area of interest was subsequently scanned at a higher magnification (water-immersion objective $\mathrm{x} 60$ with or without $\mathrm{x} 2$ zoom) by manually setting the $\mathrm{X} / \mathrm{Y}$ plane and adjusting the $\mathrm{Z}$ axis (either automatically or manually) to obtain high-resolution, clear TPLSM images. The scanning areas were $200 \times 200 \mu \mathrm{m}$ (x600) or 100x100 $\mu \mathrm{m}$ (x600 with x2 zoom) respectively. The imaging depth or imaging stack was determined arbitrarily to allow real-time three-dimensional visualization of colorectal liver metastasis in vivo. The laser power was adjusted according to the imaging depth. When imaging at larger depths, we increase the laser power level $(\leq 100 \%)$ manually using laser power level controller. To image the optimal simultaneous imaging of EGFP and RFP(DsRed2), detection sensitivity (brightness by HV) was adjusted manually for EGFP (450-500) or RFP (550-600), respectively.

Time-series imaging using intravital TPLSM. Intravital TPLSM for imaging of liver metastatic xenografts can be performed in the same mice until the formation of non-dissecting adhesions between the liver and the abdominal wall. A sodium hyaluronate and carboxymethylcellulose membrane (Seprafilm Adhesion Barrier, Genzyme Corporation) was useful to prevent the formation of postoperative adhesions between the liver and the abdominal wall. However, our preliminary experiments demonstrated that the intravital TPLSM images at four or more time points were not clear enough to observe liver metastatic xenografts because of dense fibrous adhesions. To keep the TPLSM images clear, intravital TPLSM using the same mice was performed up to three time points for time-series imaging of liver metastatic xenografts in the same mice. Precautions were also taken during the entire surgical procedure of timeseries intravital TPLSM imaging to prevent postoperative intraperitoneal infection.

The timing of intravital TPLSM imaging (Fig. 1). After inoculation of RFP-HT29 cells into the spleens of GFP nude mice, macroscopic liver metastases were observed by 8 weeks in our model. The 1st intravital TPLSM was performed at $2 \mathrm{~h}$ after inoculation to confirm the presence of RFP-HT29 cells in hepatic sinusoids, which indicate the successful inoculation. The 2nd inrtavital TPLSM was performed in the same mice to observe the liver metastatic formation and their tumor microenvironment at 6-8 weeks after inoculation. $5-\mathrm{FU}(50 \mathrm{mg} / \mathrm{kg})$ or CPT-11 $(20 \mathrm{mg} / \mathrm{kg})$ was administered intraperitoneally three times a week for three weeks after the confirmation of macroscopic liver metastases. The 3rd intravital TPLSM was also performed after the above drug administration to observe in vivo real-time chemotherapy response on the tumor microenvironment of liver metastatic xenografts in the same living mice. Thus, all mice with macroscopic liver metastases were treated with either 5-FU or CPT-11 chemotherapy. These mice were imaged at three time points by time-series intravital TPLSM. At the end of the experiments (after the 3rd intravital TPLSM), whole livers were harvested and subjected to histopathological analysis.

Immunohistochemistry for cytokeratin 20. The lack of immunological cross-reactivity with other cytokeratins means that cytokeratin 20 (CK20) has become an important tool for delineating the origin of metastatic human adenocarcinomas arising from an unknown primary source. Mouse livers were removed and fixed in $4 \%$ formaldehyde in PBS ( $\mathrm{pH} 7.4)$ for $24 \mathrm{~h}$, processed, and embedded in paraffin wax according to standard procedures. Formalin-fixed, paraffin-embedded tissue was sliced at a thickness of $3 \mu \mathrm{m}$, and the sections were placed on silane-coated slides. After deparaffinization and dehydration, the sections were autoclaved for $10 \mathrm{~min}$ in 


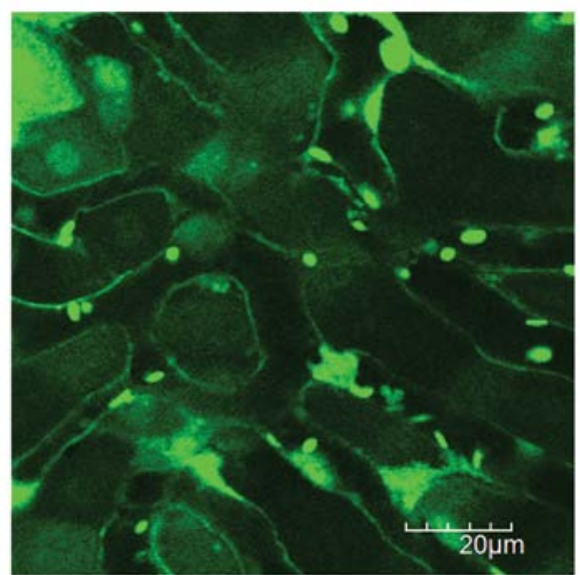

Figure 2. Normal murine liver under intravital TPLSM. Liver structures such as hepatocytes, hepatic sinusoids, hepatic endothelium, and blood cells were clearly imaged with acceptable motion artifacts in our experimental protocol (x1200). Erythrocytes were not visualized in GFP mice (19).

$10 \mathrm{mM}$ sodium citrate buffer for antigen retrieval. They were blocked and incubated with primary antibody overnight at $4^{\circ} \mathrm{C}$. Primary monoclonal anti-human CK20 antibody (Clone KBsB20.8; DakoCytomation, Denmark) was used at a dilution of 1:50 for implementation of the labeled streptavidinbiotin method (LASB2 kit/HRP, DakoCytomation). CK20 was detected using Envision reagents (Envision kit/HRP, DakoCytomation). The sections were counterstained using hematoxylin. Negative controls were run simultaneously with pre-immune immunoglobulin.

\section{Results}

A single cancer cell in hepatic sinusoids by 1st intravital TPLSM. Intravital TPLSM imaging was represented as a time-lapse two-dimensional film, and also as a z-stack three-dimensional movie from the liver surface to $\sim 100-200 \mu \mathrm{m}$ depth. The optimal imaging depth was determined arbitrarily and depended in part on the positioning of the liver lobe using the organ-stabilizing system, or on the laser power. Liver structures such as hepatocytes, hepatic sinusoids, hepatic endothelium, and blood cells such as leukocytes and platelets were clearly imaged with acceptable motion artifacts (Fig. 2). Since erythrocytes were not visualized in GFP mice (19), leukocytes were recognized as larger round cells, and platelets were recognized as smaller ones within hepatic vessels.

The 1st intravital TPLSM was performed at $2 \mathrm{~h}$ after inoculation. Initially, we identified at least five areas probably existing RFP-HT29 cells at lower magnification (x40-x100) (Fig. 3A). Next, we imaged each area of interest at higher magnification (x600) to confirm the presence of RFP-HT29 cells in hepatic sinusoids, which indicate the successful inoculation. Two hours after the inoculation of $2 \times 10^{6}$ RFP-HT29 cells to the spleen of the GFP nude mice, 5-10 RFP-HT29 cells were identified in the voxel of 210x210x100 $\mu \mathrm{m}$ (x1200) (Fig. 3B).

Liver metastatic formation by 2 nd intravital TPLSM. The preliminary experiments demonstrated that macroscopic liver metastases could be identified by 8 weeks after the inoculation of $2 \times 10^{6}$ RFP-HT2 2 cells to the spleen of GFP nude mice. Approximately $70 \%$ of all mice inoculated with RFP-HT29 cells had macroscopic liver metastatic nodules on the surface of the liver. Among them, $70 \%$ of the mice with macroscopic liver metastases could be imaged by 2 nd inrtavital TPLSM. The remaining mice with macroscopic liver metastases could not be imaged because their location is too difficult to observe in our system. The 2nd inrtavital TPLSM was performed in the same mice to observe the liver metastatic formation and their tumor microenvironment at 6-8 weeks after inoculation. There is no mouse which could not be imaged by 2 nd intravital TPLSM for dense fibrous adhesions between the liver and abdominal wall. Macroscopic liver metastatic nodules were composed of tumor cell clusters (red) and the surrounding reactive stroma with dilated/tortuous tumor vessels (green) (Fig. 4).

Tumor angiogenesis in liver metastases by 2 nd intravital TPLSM. Abnormally dilated or tortuous tumor vessels were
A

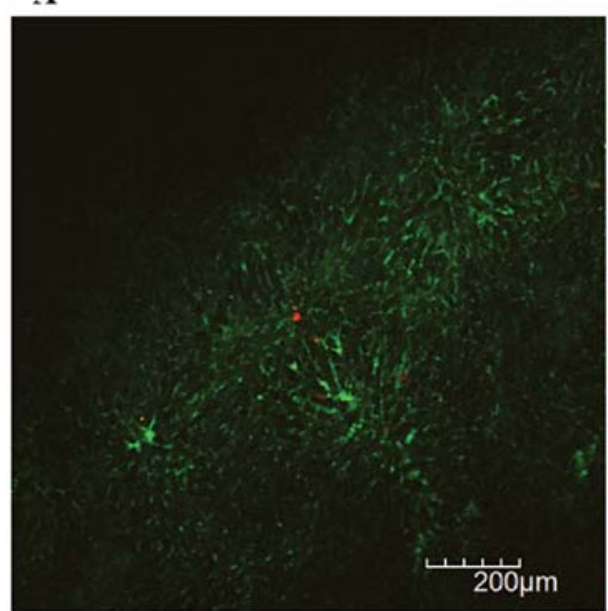

B

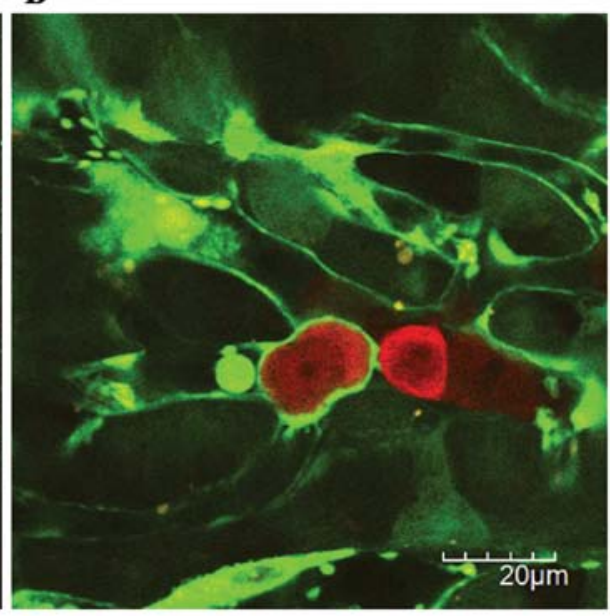

Figure 3. A single cancer cell under 1st intravital TPLSM. (A) Identification of at least five areas with probably existing RFP-HT29 cells at lower magnification (x100). (B) Two hours after inoculation, 5-10 RFP-HT29 cells were identified within hepatic sinusoids in the voxel of 210x210x100 $\mu \mathrm{m}$ (x600). 
A

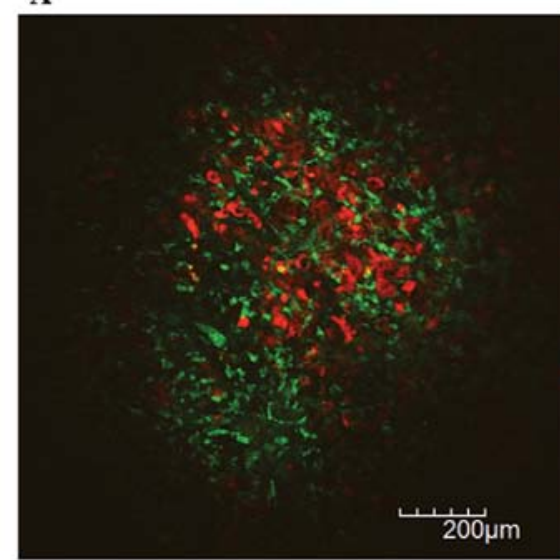

B

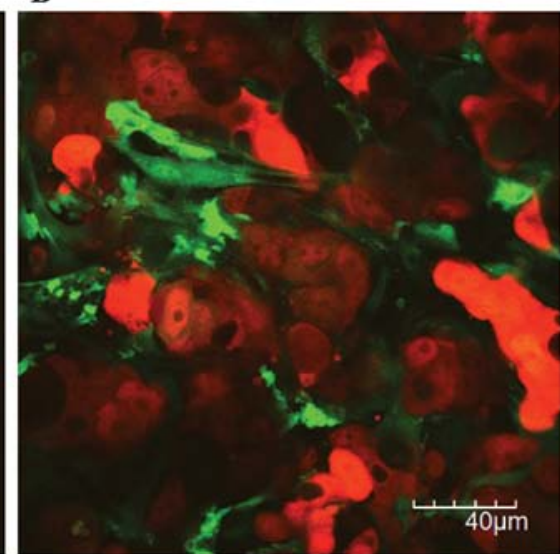

Figure 4. Liver metastatic colonization under 2nd intravital TPLSM. (A) Liver metastatic colonization at lower magnification (x100). (B) Liver metastatic colonization at higher magnification (x600). Tumor cell clusters (red) and the surrounding reactive stroma with dilated/tortuous tumor vessels (green).

A

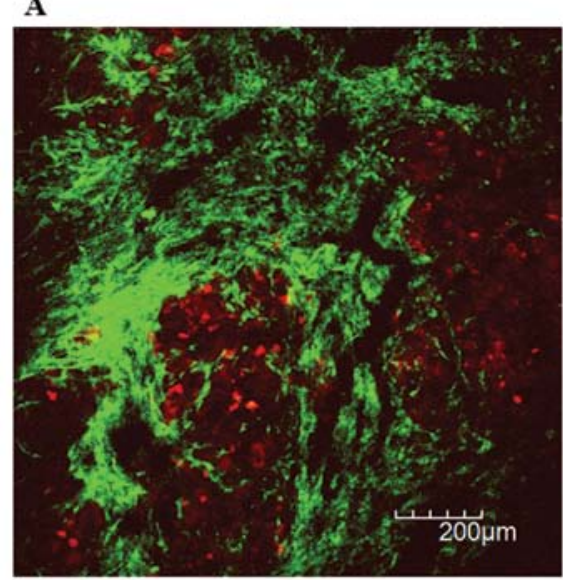

B

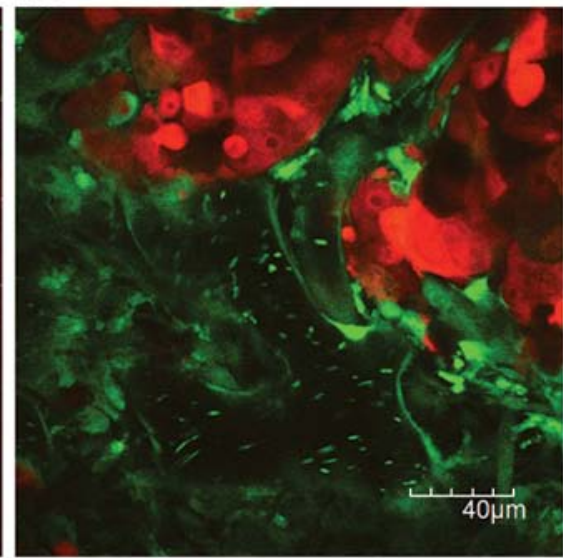

Figure 5. Tumor vessels in the liver metastases under 2nd intravital TPLSM. (A) Dilated and tortuous tumor vessels at lower magnification (x100). (B) Dilated and tortuous tumor vessels at higher magnification (x600).

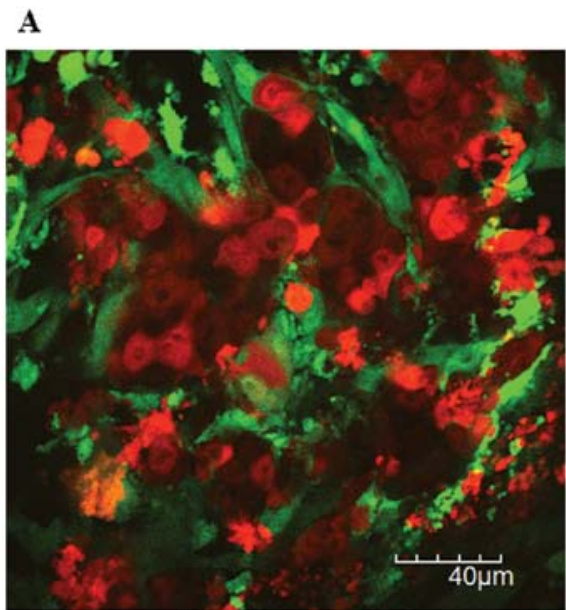

B

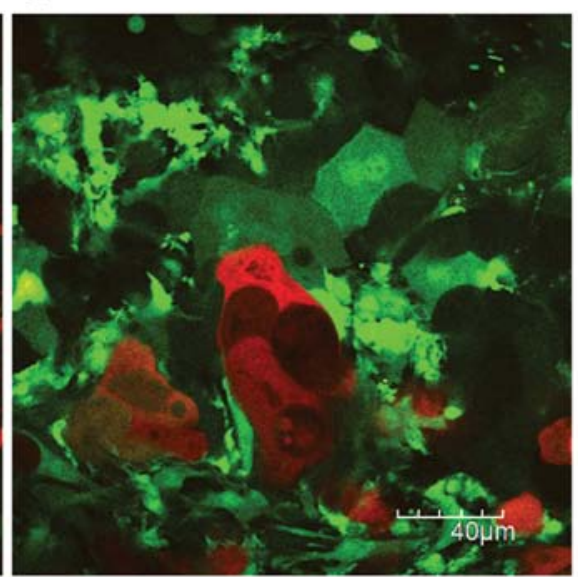

Figure 6. Treatment efficacy on liver metastatic xenografts under 3rd intravital TPLSM. With regard to the in vivo real-time chemotherapy response of metastatic tumor cells, tumor cell fragmentation and condensation (A), swelling and intracellular vacuoles (B) were observed under the 3rd intravital TPLSM.

observed in liver metastatic xenografts of RFP-HT29 cells at lower magnification (Fig. 5A). Blood cells except eryth- rocytes were clearly visualized within tumor vessels at higher magnification over x600 (Fig. 5B). The blood flow by 

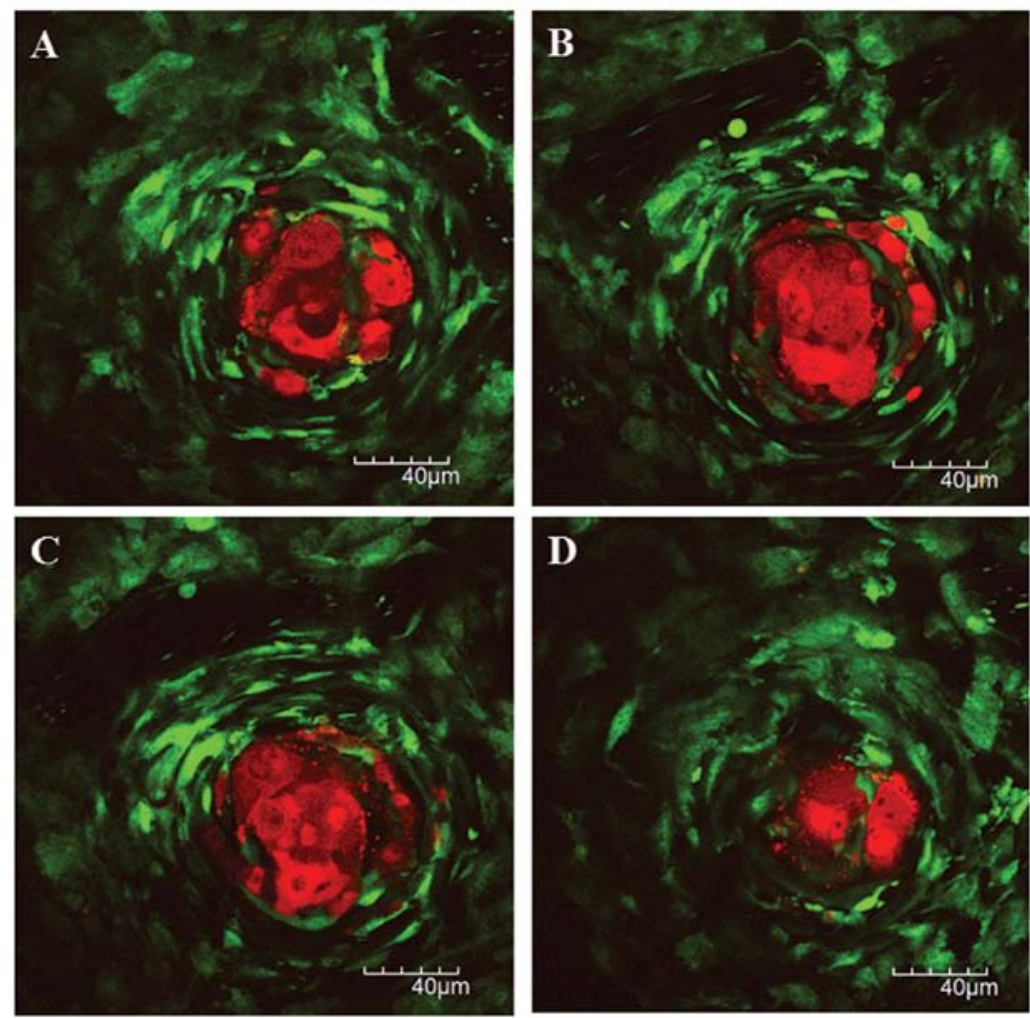

Figure 7. Peripheral tumor cell fragmentation on liver micrometases. The z-stacks sections of the micrometatic nodule were imaged from the surface (A) to the deep (D).

the movement of platelet was heterogeneous and frequently non-directional in tumor vessels of metastatic xenografts. The aggregated platelets were frequently flowing, which may indicate tumor vessel damage or intratumoral coagulation abnormality.

In vivo chemotherapy response on liver metastatic xenografts by 3rd intravital TPLSM. After the 2nd itravital TPLSM followed by 5 -FU or CPT-11 administration, the 3rd inrtavital TPLSM was performed to observe in vivo real-time chemotherapy response on the tumor microenvironment of liver metastatic xenografts in the same living mice. We imaged at least three or more mice for each treatment group which were subject to the time-series intravital TPLSM in the same mice.

With regard to the in vivo real-time chemotherapy response of metastatic tumor cells, tumor cell fragmentation, condensation, swelling and intracellular vacuoles were observed under the 3rd intravital TPLSM (Fig. 6). The smaller micrometastatic nodule was observed which showed the viable tumor cell in the central area of the nodule, the RFP-expressing fragments in the peripheral area, and the surrounding GFP-expressing host stromal cells (Fig. 7). The peripheral tumor cells may induce apoptosis by chemotherapy, resulting in the peripheral tumor cell fragmentation.

The other larger one was observed which showed the acellular central area with RFP-expressing fragments, the viable tumor cell in the peripheral area, and the surrounding GFP-expressing host stromal cells (Fig. 8). The central tumor cells may induce central tumor necrosis or apoptosis due to tumor hypoxia or chemotherapy. There was no obvious morphological difference in tumor response on liver metastatic xenografts between 5-FU and CPT-11.

Tumor vessel abnormality on liver metastatic xenografts by 3rd intravital TPLSM. With regard to the in vivo real-time chemotherapy response of host cells, the flowing of the aggregated platelets and the adhesion of them to tumor vessels were frequently observed under the 3rd intravital TPLSM. The blood flow by the platelet movement in the liver metastatic xenografts was slower than that in normal liver of the same mouse (data not shown). These results suggest that the endothelial damage or coagulation abnormality may occur within the tumor vessels after chemotherapy. The phenomena of leukocyte arrest or rolling to the endothelium was not observed within the tumor vessels of metastatic xenografts either before or after chemotherapy, as those found in the inflammatory bowel disease model.

Human CK20 immunostaining of liver metastatic xenografts after chemotherapy. Anti-human CK20 antibody was used for the detection of RFP-HT29 cells in the xenogeneic liver metastasis model. Basically, histopathological findings supported in vivo real-time observation of anticancer drug efficacy for liver metastatic xenografts by intravital TPLSM (Fig. 9A and B).

\section{Discussion}

In patients with $\mathrm{mCRC}$, the control of metastatic disease directly reflects their prognosis. Although we have now many 

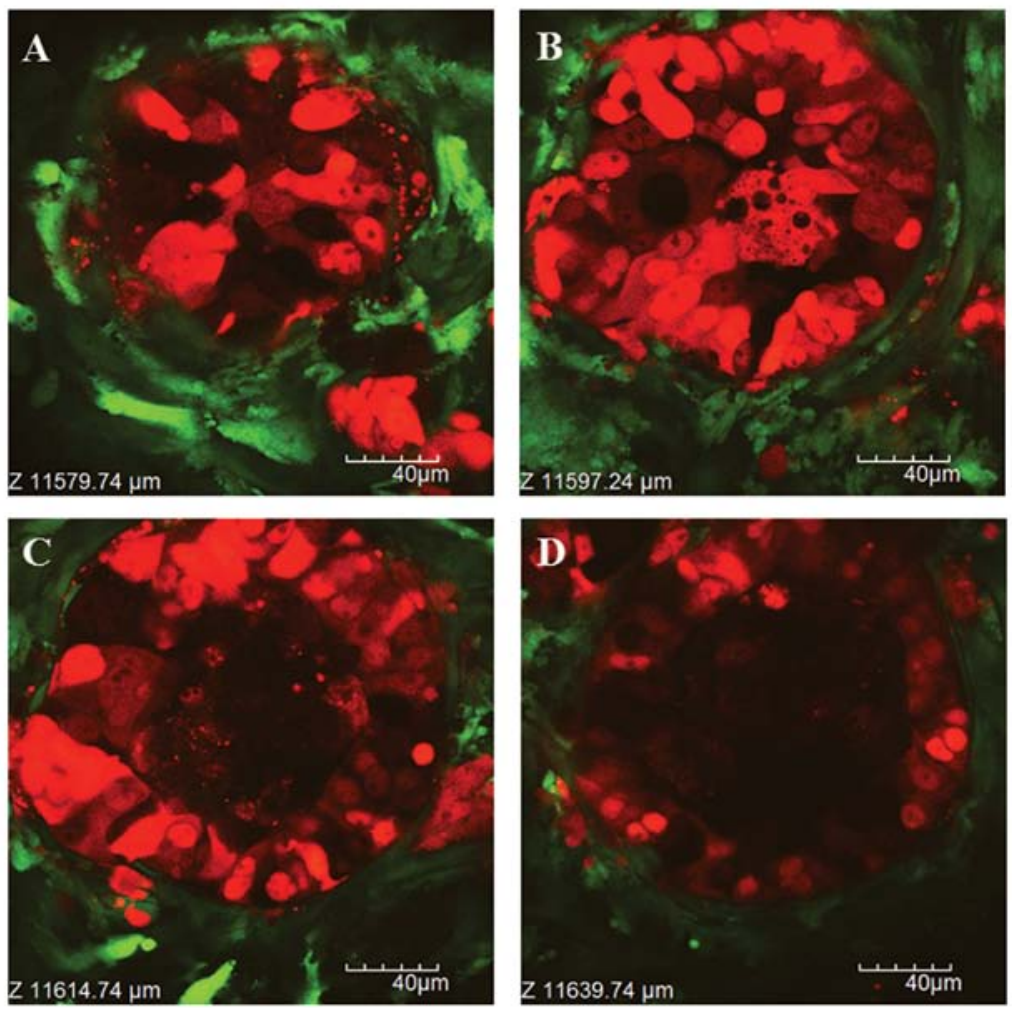

Figure 8. Central tumor cell necrosis on liver micrometases. The z-stacks sections of the micrometatic nodule were imaged from the surface (A) to the deep (D).
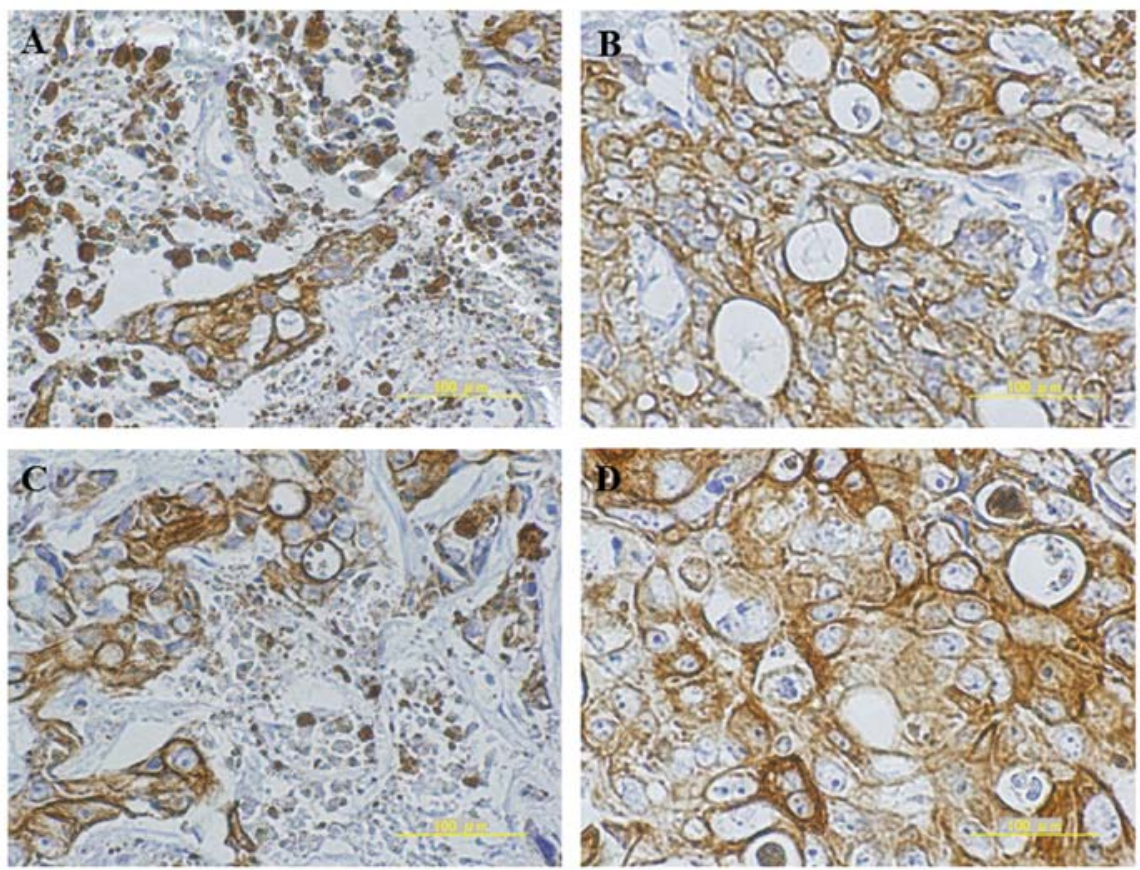

Figure 9. CK20 immunostaining of liver metastatic xenografts after chemotherapy. No obvious morphological difference was found in tumor response on liver metastatic xenografts between 5-FU and CPT-11.

types of treatment options for mCRC patients, metastatic disease control depends in part on whether or how metastatic diseases respond to chemotherapy (20). Thus, it remains particularly important to understand the mechanisms how metastatic tumor cells (and/or host stromal cells) respond or resist to chemotherapy on the metastatic organs.

Previously, we established time-series intravital TPLSM imaging of colorectal liver metastasis model using RFP 
expressing cancer cells and GFP expressing nude mice (16). We have also reported the in vivo real-time development of liver metastatic formation and tumor angiogenesis at the cellular level in the metastatic organs of the same living mice (17). In this study, we visualized the response of metastatic tumor cells to 5-FU or CPT-11 in the three-dimensional microenvironment in vivo real-time using a $\mathrm{z}$-stacks imaging. We also visualized the dynamics of host response to chemotherapy, especially platelet-endothelial interactions, in the tumor vessels of metastatic liver xenografts using a time-lapse imaging.

Chemotherapy responses such as tumor cell fragmentation, condensation, swelling and intracellular vacuoles have been observed by conventional histopathological examinations including hematoxylin-eosin or immunohistochemistry (21-23). Morphological 3D analysis can be performed by reconstructing the serial sections of the excised sample. However, we can easily image and analyze the chemotherapy response consisting of 'viable tumor cells' and 'viable stromal cells' in either twodimensionally or three-dimensionally on metastatic tumor xenografts of the living mice using our model. Although there was no obvious morphological difference in tumor response between 5-FU and CPT-11, the phenomena of peripheral and central fragmentation in the micrometastatic nodules observed in this study seem to be important on the research of cancer cell death. They may be associated with drug delivery, tumor hypoxia, and the both in the metastatic xenografts.

The platelet aggregation and the relatively decreased blood flow in the tumor vessels of colorectal liver metastases are also particular important findings. The platelet aggregation has been considered the surrogate of endothelial damage or hypercoagulable condition $(24,25)$. In this study, we did not demonstrate the direct evidence of endothelial damage such as the identification of the discontinuity of endothelium by isolectin staining. However, these finings indicate that chemotherapy may induce tumor vessel damage or intratumoral coagulation abnormality. The endothelial damage or coagulation abnormality by chemotherapy may be associated with the mechanisms of metronomic chemotherapy $(26,27)$.

Preclinically, murine xenograft models are still used for the development of new anticancer therapeutics although they are not ideal based on the criticisms voiced. There is a significant site-specific variation in response to chemotherapy for a number of human malignant tumors (28). Wilmanns et al have reported that the tumor xenografts of human colon cancer cell line, KM12L4a, at different anatomical locations showed different tumor responses to doxorubicin whose growth inhibition rate were $80 \%$ in subcutaneous xenograft, $40 \%$ in orthotopic xenograft, and $10 \%$ in liver metastatic xenograft, respectively $(29,30)$. In the preclinical model of anticancer therapeutics development, it remains an unanswered question whether the chemotherapy response of the subcutaneous (non-metastatic) tumor xenografts is representative of clinically metastatic disease which is a substantial target of chemotherapy. We believe that the metastatic tumor xenografts may be good and practical for the preclinical murine models of the evaluation of new anticancer chemotherapeutics. In this regard, time-series intravital TPLSM imaging in the same animals may be a useful tool for screening and evaluating new chemotherapeutics with less interindividual variability.
In conclusion, intravital TPLSM imaging can directly visualize the cellular morphology in tumor microenvironment in the living organs of the living animals. Time-series intravital TPLSM imaging can provide the dynamic (in vivo real-time) pathology in the same living organs of the same living animals at the indicated time points, resulting in the less interindividual variability between animals.

\section{Acknowledgements}

This study was supported by grants from the Ministry of Education, Culture, Sports, Science and Technology of Japan (KAKENHI 22591484 to K.T., 21591723 to Y.I. and 21390377 to M.K.). This work was also supported by a grant-in-aid of The Public Trust Fund for the Promotion of Surgery, Tokyo, Japan. K.T. was supported by the Okasan-Kato Foundation with regard to this study.

\section{References}

1. Gallagher DJ and Kemeny N: Metastatic colorectal cancer: from improved survival to potential cure. Oncology 78: 237-248, 2010.

2. Cunningham D, Atkin W, Lenz HJ, et al: Colorectal cancer. Lancet 375: 1030-1047, 2010.

3. Folprecht G, Gruenberger T, Bechstein WO, et al: Tumour response and secondary resectability of colorectal liver metastases following neoadjuvant chemotherapy with cetuximab: the CELIM randomised phase 2 trial. Lancet Oncol 11: 38-47, 2010.

4. Brouquet A, Abdalla EK, Kopetz S, et al: High survival rate after two-stage resection of advanced colorectal liver metastases: response-based selection and complete resection define outcome. J Clin Oncol 29: 1083-1090, 2011.

5. Tanaka K, Inoue Y and Kusunoki M: The role of cytoreduction as a multidisciplinary treatment modality for metastatic colorectal cancer. In: Colorectal Cancer: Risk, Diagnosis and Treatments. Jenkins JE (ed). Nova Science Publishers, New York, NY, pp161-176, 2010.

6. Longley DB, Harkin DP and Johnston PG: 5-Fluorouracil: mechanisms of action and clinical strategies. Nat Rev Cancer 3: 330-338, 2003.

7. $\mathrm{Xu} \mathrm{Y}$ and Villalona-Calero MA: Irinotecan: mechanisms of tumor resistance and novel strategies for modulating its activity. Ann Oncol 13: 1841-1851, 2002.

8. Raymond E, Faivre S, Chaney S, Woynarowski J and Cvitkovic E: Cellular and molecular pharmacology of oxaliplatin. Mol Cancer Ther 1: 227-235, 2002.

9. Le Dévédec SE, van Roosmalen W, Pont C, et al: Two-photon intravital multicolour imaging to study metastatic behaviour of cancer cells in vivo. Methods Mol Biol 769: 331-349, 2011.

10. Beerling E, Ritsma L, Vrisekoop N, Derksen PW and van Rheenen J: Intravital microscopy: new insights into metastasis of tumors. J Cell Sci 124: 299-310, 2011.

11. Ustione A and Piston DW: A simple introduction to multiphoton microscopy. J Microsc 243: 221-226, 2011.

12. Wang BG, König K and Halbhuber KJ: Two-photon microscopy of deep intravital tissues and its merits in clinical research. J Microsc 238: 1-20, 2010.

13. Toiyama Y, Mizoguchi A, Okugawa Y, et al: Intravital imaging of DSS-induced cecal mucosal damage in GFP-transgenic mice using two-photon microscopy. J Gastroenterol 45: 544-553, 2010.

14. Koike Y, Tanaka K, Okugawa Y, et al: In vivo real-time twophoton microscopic imaging of platelet aggregation induced by selective laser irradiation to the endothelium created in the beta-actin-green fluorescent protein transgenic mice. J Thromb Thrombolysis 32: 138-145, 2011.

15. Morimoto Y, Tanaka K, Toiyama Y, et al: Intravital threedimensional dynamic pathology of experimental colitis in living mice using two-photon laser scanning microscopy. J Gastrointest Surg 15: 1842-1850, 2011.

16. Tanaka K, Morimoto Y, Toiyama Y, et al: Intravital dual-colored visualization of colorectal liver metastasis in living mice using two photon laser scanning microscopy. Microsc Res Tech 75: 307-315, 2012. 
17. Tanaka K, Morimoto Y, Toiyama Y, et al: In vivo time-course imaging of tumor angiogenesis in colorectal liver metastases in the same living mice using two-photon laser scanning microscopy. J Oncol 2012: 265487, 2012.

18. Kawano H, Kogure T, Abe Y, Mizuno H and Miyawaki A: Two-photon dual-color imaging using fluorescent proteins. Nat Methods 5: 373-374, 2008.

19. Okabe M, Ikawa M, Kominami K, Nakanishi T and Nishimune $Y$ : 'Green mice' as a source of ubiquitous green cells. FEBS Lett 407: 313-319, 1997.

20. Small RM, Lubezky N, Shmueli E, et al: Response to chemotherapy predicts survival following resection of hepatic colo-rectal metastases in patients treated with neoadjuvant therapy. J Surg Oncol 99: 93-98, 2009.

21. Mandard AM, Dalibard F, Mandard JC, et al: Pathologic assessment of tumor regression after preoperative chemoradiotherapy of esophageal carcinoma. Clinicopathologic correlations. Cancer 73: 2680-2686, 1994.

22. Dworak O, Keilholz L and Hoffmann A: Pathological features of rectal cancer after preoperative radiochemotherapy. Int J Colorectal Dis 12: 19-23, 1997.

23. Becker K, Mueller JD, Schulmacher C, et al: Histomorphology and grading of regression in gastric carcinoma treated with neoadjuvant chemotherapy. Cancer 98: 1521-1530, 2003.
24. Furie B and Furie BC: Mechanisms of thrombus formation. N Engl J Med 359: 938-949, 2008.

25. Ruggeri ZM: Platelet adhesion under flow. Microcirculation 16: 58-83, 2009.

26. Pasquier E, Kavallaris M and André N: Metronomic chemotherapy: new rationale for new directions. Nat Rev Clin Oncol 7: 455-465, 2010.

27. Kerbel RS and Kamen BA: The anti-angiogenic basis of metronomic chemotherapy. Nat Rev Cancer 4: 423-436, 2004.

28. Talmadge JE, Singh RK, Fidler IJ and Raz A: Murine models to evaluate novel and conventional therapeutic strategies for cancer. Am J Pathol 170: 793-804, 2007.

29. Wilmanns C, Fan D, Obrian C, et al: Modulation of doxorubicin sensitivity and level of P-glycoprotein expression in human colon-carcinoma cells by ectopic and orthotopic environments in nude-mice. Int J Oncol 3: 413-422, 1993.

30. Wilmanns C, Fan D, O'Brian CA, Bucana CD and Fidler IJ: Orthotopic and ectopic organ environments differentially influence the sensitivity of murine colon carcinoma cells to doxorubicin and 5-fluorouracil. Int J Cancer 52: 98-104, 1992. 